\title{
An Efficient Mathematical Approach for the Fraction Order Differentiation Based on Future Applications of Chaotic Parameter
}

\author{
Ghulam Bary $\mathbb{D}^{1},{ }^{1}$ Waqar Ahmed $\left(\mathrm{D},{ }^{2}\right.$ Muhammad Sajid $\left(\mathbb{D},{ }^{3} \operatorname{Riaz}\right.$ Ahmad $\mathbb{D},{ }^{1}$ \\ Muhammad Farooq Saleem Khan, ${ }^{4}$ Md Fayz-Al-Asad $\mathbb{D},{ }^{5}$ \\ Nawaf N. Hamadneh (iD, ${ }^{6}$ and Ilyas Khan ${ }^{7}{ }^{7}$ \\ ${ }^{1}$ Faculty of Science, Yibin University, Yibin 644000, Sichuan, China \\ ${ }^{2}$ Department of Bionanotechnology, Hanyang University, Ansan 155-88, Republic of Korea \\ ${ }^{3}$ Faculty of Materials and Chemical Engineering, Yibin University, Yibin 644000, Sichuan, China \\ ${ }^{4}$ Faculty of International Applied Technology, Yibin University, Yibin 64400, Sichuan, China \\ ${ }^{5}$ Department of Mathematics, Bangladesh University of Engineering and Technology, Dhaka 1000, Bangladesh \\ ${ }^{6}$ Department of Basic Sciences, College of Science and Theoretical Studies, Saudi Electronic University, \\ Riyadh 11673, Saudi Arabia \\ ${ }^{7}$ Department of Mathematics, College of Science Al-Zulfi, Majmaah University, Al Majmaah 11952, Saudi Arabia
}

Correspondence should be addressed to Ghulam Bary; ghulambary@gmail.com

Received 17 August 2021; Accepted 22 September 2021; Published 16 October 2021

Academic Editor: Muhammad Shoaib Anwar

Copyright (c) 2021 Ghulam Bary et al. This is an open access article distributed under the Creative Commons Attribution License, which permits unrestricted use, distribution, and reproduction in any medium, provided the original work is properly cited.

Normalized chaotic parameters examine the characterization of the particle production fluids produced at unusual energies and investigate a remarkable behavior in quantum measurement. The analogous characterization can be analyzed to probe the chaotic systems of boson particles creating sources of extraordinary energy. We observe that the bosons appear to be the appropriate aspirants of chaos fractions, and the normalized chaotic parameters evaluate the presence of such conglomerate phases significantly. The core point of this manuscript is that we calculate and examine the normalized chaotic parameters by differential equations to analyze the characteristics of the chaotic systems and their applications in thermal as well as in mechanical engineering. With such an efficient and distinctive approach, we perceive significant consequences for the correlator at higher temperature regimes.

\section{Introduction}

The femtoscopy is extensively studied to investigate the characteristics of the chaotic and coherence systems created during the smashing of heavy nuclei at unprecedented energies [1-5]. The chaotic parameter is enormously used to extract the consequences of the hybrid systems under consideration that the boson particles are ejected usually from the partially chaotic sources to analyze the two particles interferometry. Such a unique chaotic parameter possesses the numerical values zero and one for the coherence and chaotic sources, respectively. On the other hand, it approaches unity for the chaotic emissions $[1,2,6]$. Moreover, various factors influence the lower order chaotic parameter like the misidentification of particles, laser emissions as well as the resonances of long-lived $[1,5,7,8]$. Perpetually, two particles quantum interferences are determined experimentally to investigate the heat chaotic components and the coherence characterization. It is distinguished; if the chaotic fraction is assessed more than fifty percent, then the two particles' chaotic parameter suppresses roughly twenty-five percent ratio. This is the compelling cause to probe the chaotic and coherence properties associated with the produced systems using two particles 
interferences alone [9]. Particularly, the quantum correlations for higher ranks interferences contain extra particulars which cannot be probed from the lower order interference. The experimental data about pion correlations investigation have evaluated that the reduction of chaoticity is increased appreciably at third-order correlations than that of the second-order interferences [9-11].

In particular, the femtoscopy at higher order investigates the source chaotic-coherence fractions significantly and also possesses advantages about experimental dominance by the elimination of Fourier phase and resonances decays which appeared long-lived $[6,12-16]$. The interferences of third-order measured data results are illustrated experimentally and graphically that the normalized chaotic parameters of thirdorder correlations report an obvious suppression from the given chaotic limit [15]. Such an obvious suppression of the chaos limit may occur due to the consequences of the coherence at lower temperature and momenta regimes [16]. There are multiple sources of nonchaotic emissions such as coherence of the pions and the pulse radiations which provoke the coherence of the numerous shapes [17-19].

This research work is unique because we have evaluated the chaotic and coherence properties of the thermal system with particles creating a boson gas source. It inflates relativistically by the interior region of the field potential for the special harmonic oscillator that drops out with the specific intervals of time in the hadronic phase temperature regime. Especially, the normalized chaotic parameters for thirdorder particles interferences are investigated with the applications of the one and two particles density matrices for hadronic phases at disparate temperature regimes. The nonchaotic particulars of production systems influence the chaotic parameter significantly under the considered measurements at miscellaneous temperatures and are explored to use them in thermal engineering [20-22].

This manuscript is organized into 5 sections. We investigate the correlations and chaotic parameters of differential equations in Section 2. In Section 3, we calculate and investigate the normalized chaotic parameter applications.
We analyze our model results in Section 4. Finally, in Section 5 , we summarize our findings.

\section{Correlations and Normalized Chaotic Parameters}

2.1. Two and Three Particles Correlation Functions. The two and three particles correlation functions in momentum space can be written in terms of the density matrices [21, 22] as follows:

$$
\begin{gathered}
C_{2}\left(p_{1}, p_{2}\right)=\frac{\rho^{(2)}\left(p_{1}, p_{2} ; p_{1}, p_{2}\right)}{\rho^{(1)}\left(p_{1}, p_{1}\right) \rho^{(1)}\left(p_{2}, p_{2}\right)} \\
C_{3}\left(p_{1}, p_{2}, p_{3}\right)=\frac{\rho^{(3)}\left(p_{1}, p_{2}, p_{3} ; p_{1}, p_{2}, p_{3}\right)}{\rho^{(1)}\left(p_{1}, p_{1}\right) \rho^{(1)}\left(p_{2}, p_{2}\right) \rho^{(1)}\left(p_{3}, p_{3}\right)} .
\end{gathered}
$$

Correlations in the experimental analysis of two and three particles are achieved by considering the ratio of all particles correlated and divided by the product of the uncorrelated in the momentum space:

$$
C_{2}\left(p_{1}, p_{2}\right)=1+\frac{\left|\rho^{(1)}\left(p_{1}, p_{2}\right)\right|^{2}-N_{c o}^{2}\left|U_{c o}\left(p_{1}\right)\right|^{2}\left|U_{c o}\left(p_{2}\right)\right|^{2}}{\rho^{1}\left(p_{1}, p_{1}\right) \rho^{(1)}\left(p_{2}, p_{2}\right)} \text {. }
$$

The condensation of Bose-Einstein influences the quantum correlations significantly, and the correlation functions depend crucially on the condensation. However, the effect of higher orders correlations is extensively larger than that of the lower order condensations. The condensation in the case of three particles plays a vital role during the interference of three bosons. In addition, the extraction of coherence from the experimental data possesses the several advantages during the analysis of heat exchange in the thermal and mechanical engineering $[22,23]$.

$$
\begin{aligned}
C_{3}\left(p_{1}, p_{2}, p_{3}\right)= & 1+\frac{\left|\rho^{(1)}\left(p_{1}, p_{2}\right)\right|^{2}-N_{c o}^{2}\left|U_{c o}\left(p_{1}\right)\right|^{2}\left|U_{c o}\left(p_{2}\right)\right|^{2}}{\rho^{1}\left(p_{1}, p_{1}\right) \rho^{(1)}\left(p_{2}, p_{2}\right)} \\
& +\frac{\left|\rho^{(1)}\left(p_{2}, p_{3}\right)\right|^{2}-N_{c o}^{2}\left|U_{c o}\left(p_{2}\right)\right|^{2}\left|U_{c o}\left(p_{3}\right)\right|^{2}}{\rho^{1}\left(p_{2}, p_{2}\right) \rho^{(1)}\left(p_{3}, p_{3}\right)} \\
& +\frac{\left|\rho^{(1)}\left(p_{1}, p_{3}\right)\right|^{2}-N_{c o}^{2}\left|U_{c o}\left(p_{1}\right)\right|^{2}\left|U_{c o}\left(p_{3}\right)\right|^{2}}{\rho^{1}\left(p_{1}, p_{1}\right) \rho^{(1)}\left(p_{3}, p_{3}\right)} \\
& +\frac{\rho^{(1)}\left(p_{1}, p_{2}\right) \rho^{(1)}\left(p_{2}, p_{3}\right) \rho^{(1)}\left(p_{3}, p_{1}\right)-N_{c o}^{3}\left|U_{c o}\left(p_{1}\right)\right|^{2}\left|U_{c o}\left(p_{2}\right)\right|^{2}\left|U_{c o}\left(p_{3}\right)\right|^{2}}{\rho^{1}\left(p_{1}, p_{1}\right) \rho^{(1)}\left(p_{3}, p_{3}\right) \rho^{(1)}\left(p_{2}, p_{2}\right)} \\
& +\frac{\rho^{(1)}\left(p_{2}, p_{1}\right) \rho^{(1)}\left(p_{3}, p_{2}\right) \rho^{(1)}\left(p_{1}, p_{3}\right)-N_{c o}^{3}\left|U_{c o}\left(p_{1}\right)\right|^{2}\left|U_{c o}\left(p_{2}\right)\right|^{2}\left|U_{c o}\left(p_{3}\right)\right|^{2}}{\rho^{1}\left(p_{1}, p_{1}\right) \rho^{(1)}\left(p_{3}, p_{3}\right) \rho^{(1)}\left(p_{2}, p_{2}\right)} .
\end{aligned}
$$


Here, it is more important to mention that the quantum correlation functions of higher order contained the corresponding correlators which are manipulated for two particles interferences:

$$
\begin{aligned}
& \Lambda_{2}(12)=\frac{\left|\rho^{(1)}\left(p_{1}, p_{2}\right)\right|^{2}-N_{c o}^{2}\left|U_{c o}\left(p_{1}\right)\right|^{2}\left|U_{c o}\left(p_{2}\right)\right|^{2}}{\rho^{1}\left(p_{1}, p_{1}\right) \rho^{(1)}\left(p_{2}, p_{2}\right)}, \\
& \Lambda_{2}(23)=\frac{\left|\rho^{(1)}\left(p_{2}, p_{3}\right)\right|^{2}-N_{c o}^{2}\left|U_{c o}\left(p_{2}\right)\right|^{2}\left|U_{c o}\left(p_{3}\right)\right|^{2}}{\rho^{1}\left(p_{2}, p_{3}\right) \rho^{(1)}\left(p_{3}, p_{2}\right)}, \\
& \Lambda_{2}(13)=\frac{\left|\rho^{(1)}\left(p_{1}, p_{3}\right)\right|^{2}-N_{c o}^{2}\left|U_{c o}\left(p_{1}\right)\right|^{2}\left|U_{c o}\left(p_{3}\right)\right|^{2}}{\rho^{1}\left(p_{1}, p_{1}\right) \rho^{(1)}\left(p_{3}, p_{3}\right)},
\end{aligned}
$$

where $\Lambda_{2}$ (12), $\Lambda_{2}$ (23), and $\Lambda_{2}$ (13) are the correlators of the two particles correlations which played the quantum statistical role in three particles interferences. Specifically, the genuine three particles correlator $\Lambda_{3}(123)$ can be extracted with the subtraction of lower orders correlators like two particles interferences. Thus, the mathematical expression for genuine three particle correlator can be expressed as $\Lambda_{3}(123)=C_{3}(123)-\Lambda_{2}(12)-\Lambda_{2}(23)-\Lambda_{2}(13)$.

The genuine three particles correlator possesses the peculiar properties about the behavior of chaos and coherence in the radiated system which has been copiously used in the thermal engineering. Equation (3) can be written for the true three particles correlator in order to show the importance in the field of thermal and mechanical engineering, respectively.

$$
\begin{aligned}
\Lambda_{3}(123)= & \frac{\rho^{(1)}\left(p_{1}, p_{2}\right) \rho^{(1)}\left(p_{2}, p_{3}\right) \rho^{(1)}\left(p_{3}, p_{1}\right)-N_{c o}^{3}\left|U_{c o}\left(p_{1}\right)\right|^{2}\left|U_{c o}\left(p_{2}\right)\right|^{2}\left|U_{c o}\left(p_{3}\right)\right|^{2}}{\rho^{1}\left(p_{1}, p_{1}\right) \rho^{(1)}\left(p_{3}, p_{3}\right) \rho^{(1)}\left(p_{2}, p_{2}\right)} \\
& +\frac{\rho^{(1)}\left(p_{2}, p_{1}\right) \rho^{(1)}\left(p_{3}, p_{2}\right) \rho^{(1)}\left(p_{1}, p_{3}\right)-N_{c o}^{3}\left|U_{c o}\left(p_{1}\right)\right|^{2}\left|U_{c o}\left(p_{2}\right)\right|^{2}\left|U_{c o}\left(p_{3}\right)\right|^{2}}{\rho^{1}\left(p_{1}, p_{1}\right) \rho^{(1)}\left(p_{3}, p_{3}\right) \rho^{(1)}\left(p_{2}, p_{2}\right)} .
\end{aligned}
$$

It is obvious that in the absence of the three particles quantum phases, the normalized chaos correlator $w_{3}\left(q_{3}\right)$ is numerically measured to 2 at all zero relative momenta. We have been able to proceed and check the model proposed for two as well as three particles correlations [24].

\subsection{Normalized Chaotic Parameter with Classical Methods.} The condensation of Bose-Einstein has hypercritical effect on the three particles correlations, and the exaction of such coherence from experimental data of three particles has the advantage that the phase as well as the resonance problems can be minimized by using the normalized chaotic parameter. The normalized chaotic parameter of three particle $w_{3}\left(q_{3}\right)=w_{3}\left(p_{1}, p_{2}, p_{3}\right)$ is obtained by dividing the genuine three particles correlator $\Lambda_{3}(123)$ with the square root of the product of two particles correlators:

$$
w_{3}\left(q_{3}\right)=\frac{\Lambda_{3}\left(q_{3}\right)}{\sqrt{\Lambda_{2}\left(q_{12}\right) \Lambda_{2}\left(q_{23}\right) \Lambda_{2}\left(q_{31}\right)}} .
$$

In addition, one may also isolate the Fourier phase of three particles by normalizing the genuine three particle correlation with the product of the two particles correlations:

$$
w_{3}\left(q_{3}\right)=\frac{\left[c_{3}\left(q_{3}\right)-1\right]}{\sqrt{\left[c_{2}\left(q_{12}\right)-1\right]\left[c_{2}\left(q_{23}\right)-1\right]\left[c_{2}\left(q_{13}\right)-1\right]}} .
$$

It has been mentioned that the genuine three particles correlator can be expressed in terms of the momentumdependent density matrices given as follows:

$$
\begin{aligned}
R_{3}(123)= & 2 . \rho^{(1)}\left(p_{1}, p_{2}\right) \rho^{(1)}\left(p_{2}, p_{3}\right) \rho^{(1)}\left(p_{3}, p_{1}\right) \\
& {\left[1-g\left(p_{1}, p_{2}, p_{3}\right)\right], }
\end{aligned}
$$

where

$$
g\left(p_{1}, p_{2}, p_{3}\right)=\frac{N_{c o}^{3}\left|U_{c o}\left(p_{1}\right)\right|^{2}\left|U_{c o}\left(p_{2}\right)\right|^{2}\left|U_{c o}\left(p_{3}\right)\right|^{2}}{\rho^{(1)}\left(p_{1}, p_{1}\right) \rho^{(1)}\left(p_{2}, p_{2}\right) \rho^{(1)}\left(p_{3}, p_{3}\right)} .
$$

In the aforementioned equations, $N_{c o}$ and $U_{c o}(p)$ express the particles number and the corresponding wave functions in the ground state. Moreover, the product of the two particles correlators for the quantum statistical interference can be manipulated as

$$
\Lambda_{2}(12) \Lambda_{2}(23) \Lambda_{2}(13)=\rho^{(1)}\left(p_{1}, p_{2}\right) \rho^{(1)}\left(p_{2}, p_{3}\right) \rho^{(1)}\left(p_{1}, p_{3}\right) \times P
$$

It is crucial to mention that the multiplier factor $P$ represents the square root of the product of the three particles correlators, and it can be expressed as $\sqrt{\left[1-g\left(p_{1}, p_{2}\right)\right]\left[1-g\left(p_{2}, p_{3}\right)\right]\left[1-g\left(p_{1}, p_{3}\right)\right]}$. Moreover, the relative coherence probability can be measured by using the partially coherence probability to the total probability during the emissions of particles: 


$$
\begin{aligned}
& g\left(p_{1}, p_{2}\right)=\frac{N_{c o}^{2}\left|U_{c o}\left(p_{1}\right)\right|^{2}\left|U_{c o}\left(p_{2}\right)\right|^{2}}{\left|\rho^{(1)}\left(p_{1}, p_{2}\right)\right|^{2}}, \\
& g\left(p_{2}, p_{3}\right)=\frac{N_{c o}^{2}\left|U_{c o}\left(p_{2}\right)\right|^{2}\left|U_{c o}\left(p_{3}\right)\right|^{2}}{\left|\rho^{(1)}\left(p_{2}, p_{3}\right)\right|^{2}}, \\
& g\left(p_{1}, p_{3}\right)=\frac{N_{c o}^{2}\left|U_{c o}\left(p_{1}\right)\right|^{2}\left|U_{c o}\left(p_{3}\right)\right|^{2}}{\left|\rho^{(1)}\left(p_{1}, p_{3}\right)\right|^{2}} .
\end{aligned}
$$

In particular, when we substitute the values of equations (8) and (10) into equation (6) under the important assumption that the emissions of three particles possess the identical momenta $p_{1}=p_{2}=p_{3}=p$, then the corresponding results can be written as

$$
\begin{gathered}
g(p, p, p)=\frac{N_{c o}^{3}\left|U_{c o}(p)\right|^{6}}{\left|\rho^{(1)}(p)\right|^{3}}=\frac{\left|\rho_{c o}(p)\right|^{3}}{|\rho(p)|^{3}}, \\
g(p, p)=\frac{N_{c o}^{2}\left|U_{c o}(p)\right|^{4}}{\left|\rho^{(1)}(p, p)\right|^{2}}=\frac{\left|\rho_{c o}(p)\right|^{2}}{|\rho(p)|^{2}} .
\end{gathered}
$$

The genuine three particles correlator in the higher correlations contains the excessive information about the particle ejecting sources, and it acquired the sophisticated form with the substitution of equation (12) into equation (8):

$$
\Lambda_{3}\left(q_{3}\right)=2 . \rho^{(1)}(p, p) \rho^{(1)}(p, p) \rho^{(1)}(p, p)\left[1-\left[\frac{\rho_{c o}(p)}{\rho(p)}\right]^{3}\right] .
$$

In addition, the product of the two particles correlator acquires the fruitful results when we use equation (18) into equation (13) for the three particles ejection. Such useful results can be obtained by using the difference equations, and the application of the analytical techniques become

$$
\begin{aligned}
& \Lambda_{2}(12) \Lambda_{2}(23) \Lambda_{2}(13)=\rho^{(1)}(p, p) \rho^{(1)} \\
& (p, p) \rho^{(1)}(p, p) \sqrt{\left[1-\left[\frac{\rho_{c o}(p)}{\rho(p)}\right]^{2}\right]^{3}} .
\end{aligned}
$$

Consequently, the results of normalized three particles chaotic parameter possess the sophistical shape after the eradication of some unusual terms, and it can be happen when we substitute the values of equations (14) and (15) into equation (6):

$$
w_{3}(p)=2 \frac{\left[1-\left[\rho_{c o}(p) / \rho^{(p)}\right]^{3}\right]}{\sqrt{\left[1-\left[\rho_{c o}(p) / \rho^{(p)}\right]^{2}\right]^{3}}} .
$$

The sensitive parameter of the coherence fraction $D(p)=\rho_{c o}(p) / \rho(p)$ and the expression for normalized three particles chaotic parameter can be written in terms of coherence fraction as follows:

$$
w_{3}(p)=2 \frac{\left[1-[D(p)]^{3}\right]}{\left[1-[D(p)]^{2}\right]^{3 / 2}} .
$$

In particular, the chaotic parameter $w_{3}(p)$ indicates that the value of correlator becomes 2 for the complete chaotic sources but it reflects to infinity for complete coherence sources. The results of $w_{3}(p)$ may not explain the experimental data of chaos fraction. Here, the question is that what can we do to explain the experimental data in order to examine the chaos fraction? We can achieve our goal to modify the old model in order to use the new techniques which compelled us to study the higher order quantum correlations in particular direction $[24,25]$.

\section{Characteristics of Normalized Chaotic Parameter}

The correlations of two particles are untrammelled about FT phase but it persists in the correlations of three particles considerably. However, such kind of considered phase is potentially isolated by weighing up the cumulant interferences of three particles cumulant to two particles correlations. It is prevailed by the division of three particles correlator under root of computed two particles correlators. Now, we have been able to express the normalized chaotic three particles correlator for aforementioned sources by using the expressions of two as well as three particles correlators. It has been observed that the systems of particles emission behave partially chaotic, and the density matrix for such systems possess the partially coherence as well as partially chaotic components. The mathematical expression for the particle emitted fluid can be illustrated as the sum of the chaotic and coherence components [25-28]:

$$
\rho^{(1)}\left(p_{1}, p_{2}\right)=\rho_{c h}^{(1)}\left(p_{1}, p_{2}\right)+\rho_{c o}^{(1)}\left(p_{1}, p_{2}\right),
$$

where $\rho_{c h}^{(1)}\left(p_{1}, p_{2}\right)$ represents the density matrix for the chaotic component of the particles ejected sources and $\rho_{c o}^{(1)}\left(p_{1}, p_{2}\right)$ shows the one particle density matrix of the corresponding coherence component. It is more crucial to mention that the density matrices at higher order can be written in terms of two particles density matrices in order to calculate the correlation functions at higher order. We can 
expand our mathematical expression of two particles interferences into three particles quantum correlations sophistically given as follows:

$$
\begin{aligned}
\rho^{(1)}\left(p_{1}, p_{2}\right) \times \rho^{(1)}\left(p_{2}, p_{3}\right) \times \rho^{(1)}\left(p_{1}, p_{3}\right)= & {\left[\rho_{c h}^{(1)}\left(p_{1}, p_{2}\right)+\rho_{c o}^{(1)}\left(p_{1}, p_{2}\right)\right] } \\
& \times\left[\rho_{c h}^{(1)}\left(p_{2}, p_{3}\right)+\rho_{c o}^{(1)}\left(p_{2}, p_{3}\right)\right] \\
& \times\left[\rho_{c h}^{(1)}\left(p_{1}, p_{3}\right)+\rho_{c o}^{(1)}\left(p_{1}, p_{3}\right)\right] .
\end{aligned}
$$

These density matrices can be multiplied in order to obtain the normalized chaotic parameter, and the adopted methodology of differential equations leads for the multiplication purposes:

$$
\begin{aligned}
\rho^{(1)}\left(p_{1}, p_{2}\right) \times \rho^{(1)}\left(p_{2}, p_{3}\right) \times \rho^{(1)}\left(p_{1}, p_{3}\right)= & \rho_{c h}^{(1)}\left(p_{1}, p_{2}\right) \cdot \rho_{c h}^{(1)}\left(p_{2}, p_{3}\right) \cdot \rho_{c h}^{(1)}\left(p_{1}, p_{3}\right) \\
& +\rho_{c h}^{(1)}\left(p_{1}, p_{2}\right) \cdot \rho_{c h}^{(1)}\left(p_{2}, p_{3}\right) \cdot \rho_{c o}^{(1)}\left(p_{1}, p_{3}\right) \\
& +\rho_{c h}^{(1)}\left(p_{1}, p_{2}\right) \cdot \rho_{c o}^{(1)}\left(p_{2}, p_{3}\right) \cdot \rho_{c h}^{(1)}\left(p_{1}, p_{3}\right) \\
& +\rho_{c o}^{(1)}\left(p_{1}, p_{2}\right) \cdot \rho_{c h}^{(1)}\left(p_{2}, p_{3}\right) \cdot \rho_{c h}^{(1)}\left(p_{1}, p_{3}\right) \\
& +\rho_{c h}^{(1)}\left(p_{1}, p_{2}\right) \cdot \rho_{c o}^{(1)}\left(p_{2}, p_{3}\right) \cdot \rho_{c o}^{(1)}\left(p_{1}, p_{3}\right) \\
& +\rho_{c o}^{(1)}\left(p_{1}, p_{2}\right) \cdot \rho_{c h}^{(1)}\left(p_{2}, p_{3}\right) \cdot \rho_{c o}^{(1)}\left(p_{1}, p_{3}\right) \\
& +\rho_{c o}^{(1)}\left(p_{1}, p_{2}\right) \cdot \rho_{c o}^{(1)}\left(p_{2}, p_{3}\right) \cdot \rho_{c h}^{(1)}\left(p_{1}, p_{3}\right) \\
& +\rho_{c o}^{(1)}\left(p_{1}, p_{2}\right) \cdot \rho_{c o}^{(1)}\left(p_{2}, p_{3}\right) \cdot \rho_{c o}^{(1)}\left(p_{1}, p_{3}\right) .
\end{aligned}
$$

In particular, the possessions of the quantum statistical correlations can be exhibited for the symmetrization of the particles emission points. It has been substantiated that the symmetrization only exists for independently ejected boson particles, and such effect is missing for the coherence particle creations $[29,30]$. It has been noted that the productions of bosons from the coherence state behave collectively, and the symmetrization does not occur for pairs of the ejected coherence bosons. Such phenomena can also appear for the mixed pairs of particles from the coherence pool and the other from the chaotic pools of particles production. Thus, the required density matrix can be expressed in terms of the lower energy ground state wave function:

$$
\begin{aligned}
\rho_{c o}^{(1)}\left(p_{1}, p_{2}\right) & =N_{c o} U_{c o}\left(p_{1}\right) U_{c o}\left(p_{2}\right) \\
\rho_{c o}^{(1)}\left(p_{2}, p_{3}\right) & =N_{c o} U_{c o}\left(p_{2}\right) U_{c o}\left(p_{3}\right) \\
\rho_{c o}^{(1)}\left(p_{1}, p_{3}\right) & =N_{c o} U_{c o}\left(p_{1}\right) U_{c o}\left(p_{3}\right) \\
\rho_{c o}^{(1)}\left(p_{1}, p_{2}\right) \cdot \rho_{c o}^{(1)}\left(p_{2}, p_{3}\right) & =N_{c o}^{2} U_{c o}\left(p_{1}\right) U_{c o}\left(p_{3}\right)\left|U_{c o}\left(p_{2}\right)\right|^{2} \\
\rho_{c o}^{(1)}\left(p_{1}, p_{2}\right) \cdot \rho_{c o}^{(1)}\left(p_{1}, p_{3}\right) & =N_{c o}^{2}\left|U_{c o}\left(p_{1}\right)\right|^{2} U_{c o}\left(p_{2}\right) U_{c o}\left(p_{3}\right) \\
\rho_{c o}^{(1)}\left(p_{2}, p_{3}\right) \cdot \rho_{c o}^{(1)}\left(p_{1}, p_{3}\right) & =N_{c o}^{2} U_{c o}\left(p_{1}\right) U_{c o}\left(p_{2}\right)\left|U_{c o}\left(p_{3}\right)\right|^{2},
\end{aligned}
$$

where $N_{c o}$ and $U_{c o}$ represent the coherence particles number and their associated coherence wave functions, respectively. Here, the above expressions can be manipulated for the further calculations as follows:

$$
\begin{aligned}
& \rho_{c o}^{(1)}\left(p_{1}, p_{2}\right) \cdot \rho_{c o}^{(1)}\left(p_{2}, p_{3}\right) \cdot \rho_{c o}^{(1)}\left(p_{1}, p_{3}\right) \\
& =N_{c o}^{3}\left|U_{c o}\left(p_{1}\right) U_{c o}\left(p_{2}\right) U_{c o}\left(p_{3}\right)\right|^{2} .
\end{aligned}
$$

Furthermore, the density matrix for the chaotic emissions can be written in the form of the coherence density components as well as the total density matrix by using the following equation:

$$
\begin{aligned}
& \rho_{c h}^{(1)}\left(p_{1}, p_{2}\right)=\rho^{(1)}\left(p_{1}, p_{2}\right)-\rho_{c o}^{(1)}\left(p_{1}, p_{2}\right), \\
& \rho_{c h}^{(1)}\left(p_{2}, p_{3}\right)=\rho^{(1)}\left(p_{2}, p_{3}\right)-\rho_{c o}^{(1)}\left(p_{2}, p_{3}\right), \\
& \rho_{c h}^{(1)}\left(p_{1}, p_{3}\right)=\rho^{(1)}\left(p_{1}, p_{3}\right)-\rho_{c o}^{(1)}\left(p_{1}, p_{3}\right) .
\end{aligned}
$$

Specifically, the emissions of three particles illustrate in such a way that the two density matrices for coherence emissions and one for the chaotic emission. These results can be expressed in terms of the wave functions: 


$$
\begin{aligned}
\rho_{c h}^{(1)}\left(p_{1}, p_{2}\right) \cdot \rho_{c o}^{(1)}\left(p_{2}, p_{3}\right) \cdot \rho_{c o}^{(1)}\left(p_{1}, p_{3}\right)= & {\left[\rho^{(1)}\left(p_{1}, p_{2}\right)-\rho_{c o}^{(1)}\left(p_{1}, p_{2}\right)\right] } \\
& \times\left[N_{c o}^{2} U_{c o}\left(p_{1}\right) U_{c o}\left(p_{2}\right)\left|U_{c o}\left(p_{3}\right)\right|^{2}\right] .
\end{aligned}
$$

The aforementioned equations can be transformed into the symmetrical shape to perform the basic multiplications, and such expression can be expressed in terms of nonchaotic emission:

$$
\begin{aligned}
\rho_{c h}^{(1)}\left(p_{1}, p_{2}\right) \cdot \rho_{c o}^{(1)}\left(p_{2}, p_{3}\right) \cdot \rho_{c o}^{(1)}\left(p_{1}, p_{3}\right)= & \rho^{(1)}\left(p_{1}, p_{2}\right) N_{c o}^{2} U_{c o}\left(p_{1}\right) U_{c o}\left(p_{2}\right)\left|U_{c o}\left(p_{3}\right)\right|^{2} \\
& -\left.N_{c o}^{3}\left|U_{c o}\left(p_{1}\right)\right| U_{c o}\left(p_{2}\right) U_{c o}\left(p_{3}\right)\right|^{2} .
\end{aligned}
$$

On the other hand, the density matrices for the symmetrization of the other triplet manipulated in the form of nonchaotic emissions are as follows:

$$
\begin{aligned}
\rho_{c h}^{(1)}\left(p_{2}, p_{3}\right) \cdot \rho_{c o}^{(1)}\left(p_{1}, p_{2}\right) \cdot \rho_{c o}^{(1)}\left(p_{1}, p_{3}\right)= & N_{c o}^{2} \rho^{(1)}\left(p_{2}, p_{3}\right) U_{c o}\left(p_{2}\right) U_{c o}\left(p_{3}\right)\left|U_{c o}\left(p_{1}\right)\right|^{2} \\
& -\left.N_{c o}^{3}\left|U_{c o}\left(p_{1}\right)\right| U_{c o}\left(p_{2}\right) U_{c o}\left(p_{3}\right)\right|^{2} .
\end{aligned}
$$

Similarly, the density matrices for the chaotic and coherent particles emissions can also be expressed as follows:

$$
\begin{aligned}
\rho_{c h}^{(1)}\left(p_{1}, p_{3}\right) \cdot \rho_{c o}^{(1)}\left(p_{1}, p_{2}\right) \cdot \rho_{c o}^{(1)}\left(p_{1}, p_{3}\right)= & N_{c o}^{2} \rho^{(1)}\left(p_{1}, p_{3}\right) U_{c o}\left(p_{1}\right) U_{c o}\left(p_{3}\right)\left|U_{c o}\left(p_{2}\right)\right|^{2} \\
& -\left.N_{c o}^{3}\left|U_{c o}\left(p_{1}\right)\right| U_{c o}\left(p_{2}\right) U_{c o}\left(p_{3}\right)\right|^{2} .
\end{aligned}
$$

We are able to generalize the density distributions method to examine the solutions of the chaos fraction by using the differential equations. Different techniques of such fractional differential equations play a vital role in order to probe the types of chaos, coherence, and implicit solutions $[31,32]$.

In addition, we substitute the mentioned values in equation (20) in order to adopt the proper mathematical expression in sophisticated shape:

$$
\begin{aligned}
\rho^{(1)}\left(p_{1}, p_{2}\right) \cdot \rho^{(1)}\left(p_{2}, p_{3}\right) \cdot \rho^{(1)}\left(p_{1}, p_{3}\right)= & \rho_{c h}^{(1)}\left(p_{1}, p_{2}\right) \cdot \rho_{c h}^{(1)}\left(p_{2}, p_{3}\right) \cdot \rho_{c h}^{(1)}\left(p_{1}, p_{3}\right) \\
& +\rho_{c h}^{(1)}\left(p_{1}, p_{2}\right) \cdot \rho_{c h}^{(1)}\left(p_{2}, p_{3}\right) \cdot \rho_{c o}^{(1)}\left(p_{1}, p_{3}\right) \\
& +\rho_{c h}^{(1)}\left(p_{1}, p_{2}\right) \cdot \rho_{c o}^{(1)}\left(p_{2}, p_{3}\right) \cdot \rho_{c h}^{(1)}\left(p_{1}, p_{3}\right) \\
& +\rho_{c o}^{(1)}\left(p_{1}, p_{2}\right) \cdot \rho_{c h}^{(1)}\left(p_{2}, p_{3}\right) \cdot \rho_{c h}^{(1)}\left(p_{1}, p_{3}\right) \\
& +\rho^{(1)}\left(p_{1}, p_{2}\right) N_{c o}^{2} U_{c o}\left(p_{1}\right) U_{c o}\left(p_{2}\right)\left|U_{c o}\left(p_{3}\right)\right|^{2} \\
& -N_{c o}^{3}\left|U_{c o}\left(p_{1}\right) U_{c o}\left(p_{2}\right) U_{c o}\left(p_{3}\right)\right|^{2} \\
& +\rho^{(1)}\left(p_{2}, p_{3}\right) N_{c o}^{2} U_{c o}\left(p_{2}\right) U_{c o}\left(p_{3}\right)\left|U_{c o}\left(p_{1}\right)\right|^{2} \\
& -N_{c o}^{3}\left|U_{c o}\left(p_{1}\right) U_{c o}\left(p_{2}\right) U_{c o}\left(p_{3}\right)\right|^{2}
\end{aligned}
$$




$$
\begin{aligned}
& +\rho^{(1)}\left(p_{1}, p_{3}\right) N_{c o}^{2} U_{c o}\left(p_{1}\right) U_{c o}\left(p_{3}\right)\left|U_{c o}\left(p_{2}\right)\right|^{2} \\
& -N_{c o}^{3}\left|U_{c o}\left(p_{1}\right) U_{c o}\left(p_{2}\right) U_{c o}\left(p_{3}\right)\right|^{2} \\
& +N_{c o}^{3}\left|U_{c o}\left(p_{1}\right) U_{c o}\left(p_{2}\right) U_{c o}\left(p_{3}\right)\right|^{2}, \\
\rho^{(1)}\left(p_{1}, p_{2}\right) \cdot \rho^{(1)}\left(p_{2}, p_{3}\right) \cdot \rho^{(1)}\left(p_{1}, p_{3}\right)= & \rho_{c h}^{(1)}\left(p_{1}, p_{2}\right) \cdot \rho_{c h}^{(1)}\left(p_{2}, p_{3}\right) \cdot \rho_{c h}^{(1)}\left(p_{1}, p_{3}\right) \\
& +\rho_{c h}^{(1)}\left(p_{1}, p_{2}\right) \cdot \rho_{c h}^{(1)}\left(p_{2}, p_{3}\right) \cdot \rho_{c o}^{(1)}\left(p_{1}, p_{3}\right) \\
& +\rho_{c h}^{(1)}\left(p_{1}, p_{2}\right) \cdot \rho_{c o}^{(1)}\left(p_{2}, p_{3}\right) \cdot \rho_{c h}^{(1)}\left(p_{1}, p_{3}\right) \\
& +\rho_{c o}^{(1)}\left(p_{1}, p_{2}\right) \cdot \rho_{c h}^{(1)}\left(p_{2}, p_{3}\right) \cdot \rho_{c h}^{(1)}\left(p_{1}, p_{3}\right) \\
& +\rho^{(1)}\left(p_{1}, p_{2}\right) N_{c o}^{2} U_{c o}\left(p_{1}\right) U_{c o}\left(p_{2}\right)\left|U_{c o}\left(p_{3}\right)\right|^{2} \\
& -2 N_{c o}^{3}\left|U_{c o}\left(p_{1}\right) U_{c o}\left(p_{2}\right) U_{c o}\left(p_{3}\right)\right|^{2} \\
& +\rho^{(1)}\left(p_{2}, p_{3}\right) N_{c o}^{2} U_{c o}\left(p_{2}\right) U_{c o}\left(p_{3}\right)\left|U_{c o}\left(p_{1}\right)\right|^{2} \\
& +\rho^{(1)}\left(p_{1}, p_{3}\right) N_{c o}^{2} U_{c o}\left(p_{1}\right) U_{c o}\left(p_{3}\right)\left|U_{c o}\left(p_{2}\right)\right|^{2} .
\end{aligned}
$$

The possible terms can be represented for the explanation of the experimental data which play a vital role in the quantum interferences, and these selected components are expressed as follows:

$$
\begin{aligned}
\rho^{(1)} & \left(p_{1}, p_{2}\right) \rho^{(1)}\left(p_{2}, p_{3}\right) \cdot \rho^{(1)}\left(p_{1}, p_{3}\right)+2 N_{c o}^{3}\left|U_{c o}\left(p_{1}\right) U_{c o}\left(p_{2}\right) U_{c o}\left(p_{3}\right)\right|^{2} \\
& -\rho^{(1)}\left(p_{1}, p_{2}\right) N_{c o}^{2} U_{c o}\left(p_{1}\right) U_{c o}\left(p_{2}\right)\left|U_{c o}\left(p_{3}\right)\right|^{2} \\
& -\rho^{(1)}\left(p_{2}, p_{3}\right) N_{c o}^{2} U_{c o}\left(p_{2}\right) U_{c o}\left(p_{3}\right)\left|U_{c o}\left(p_{1}\right)\right|^{2} \\
& -\rho^{(1)}\left(p_{1}, p_{3}\right) N_{c o}^{2} U_{c o}\left(p_{1}\right) U_{c o}\left(p_{3}\right)\left|U_{c o}\left(p_{2}\right)\right|^{2} \\
= & {\left[\rho_{c h}^{(1)}\left(p_{1}, p_{2}\right) \cdot \rho_{c h}^{(1)}\left(p_{2}, p_{3}\right) \cdot \rho_{c h}^{(1)}\left(p_{1}, p_{3}\right)\right] } \\
& +\rho_{c h}^{(1)}\left(p_{1}, p_{2}\right) \cdot \rho_{c h}^{(1)}\left(p_{2}, p_{3}\right) \cdot \rho_{c o}^{(1)}\left(p_{1}, p_{3}\right) \\
& +\rho_{c h}^{(1)}\left(p_{1}, p_{2}\right) \cdot \rho_{c o}^{(1)}\left(p_{2}, p_{3}\right) \cdot \rho_{c h}^{(1)}\left(p_{1}, p_{3}\right) \\
& +\rho_{c o}^{(1)}\left(p_{1}, p_{2}\right) \cdot \rho_{c h}^{(1)}\left(p_{2}, p_{3}\right) \cdot \rho_{c h}^{(1)}\left(p_{1}, p_{3}\right) .
\end{aligned}
$$

Basically chaotic fluid is utilized to enhance the thermal nature in the corresponding equipment such as heat exchanger, electronic, and nuclear systems. The transfer of energy, momentum, and heat in the partially chaotic fluids has been discussed with the density matrices by several researchers in order to control the aforementioned phenomena [33-35]. It is obvious that the source of ejected particles may be the pure chaotic or partially coherence but this equation plays a versatile role in all considerable cases. We can check the acquired equation for normalized three particles chaotic parameter (correlator) whether it follows the experimental results to examine the chaotic and coherence fractions of the particles ejected sources. Particularly, the three particles correlator can be written in the meaningful way when we use the expression of equations (29) and (5), respectively. 


$$
\begin{aligned}
\Lambda_{3}(123)= & 2\left[\rho^{(1)}\left(p_{1}, p_{2}\right) \cdot \rho^{(1)}\left(p_{2}, p_{3}\right) \cdot \rho^{(1)}\left(p_{1}, p_{3}\right)+2 N_{c o}^{3}\left|U_{c o}\left(p_{1}\right) U_{c o}\left(p_{2}\right) U_{c o}\left(p_{3}\right)\right|^{2}\right. \\
& -\rho^{(1)}\left(p_{1}, p_{2}\right) N_{c o}^{2} U_{c o}\left(p_{1}\right) U_{c o}\left(p_{2}\right)\left|U_{c o}\left(p_{3}\right)\right|^{2}-\rho^{(1)}\left(p_{2}, p_{3}\right) N_{c o}^{2} U_{c o}\left(p_{2}\right) U_{c o}\left(p_{3}\right)\left|U_{c o}\left(p_{1}\right)\right|^{2} \\
& \left.-\rho^{(1)}\left(p_{1}, p_{3}\right) N_{c o}^{2} U_{c o}\left(p_{1}\right) U_{c o}\left(p_{3}\right)\left|U_{c o}\left(p_{2}\right)\right|^{2}\right], \\
\Lambda_{3}(123)= & 2 \cdot \rho^{(1)}\left(p_{1}, p_{2}\right) \cdot \rho^{(1)}\left(p_{2}, p_{3}\right) \cdot \rho^{(1)}\left(p_{3}, p_{1}\right) \\
& \cdot\left[1+2 \frac{N_{c o}^{3}\left|U_{c o}\left(p_{1}\right)\right|^{2}\left|U_{c o}\left(p_{2}\right)\right|^{2}\left|U_{c o}\left(p_{3}\right)\right|^{2}}{\rho^{1}\left(p_{1}, p_{1}\right) \rho^{(1)}\left(p_{3}, p_{3}\right) \rho^{(1)}\left(p_{2}, p_{2}\right)}-\frac{\rho^{(1)}\left(p_{1}, p_{2}\right) N_{c o}^{2} U_{c o}\left(p_{1}\right) U_{c o}\left(p_{2}\right)\left|U_{c o}\left(p_{3}\right)\right|^{2}}{\rho^{1}\left(p_{1}, p_{1}\right) \rho^{(1)}\left(p_{3}, p_{3}\right) \rho^{(1)}\left(p_{2}, p_{2}\right)}\right. \\
& \left.-\frac{\rho^{(1)}\left(p_{2}, p_{3}\right) N_{c o}^{2} U_{c o}\left(p_{2}\right) U_{c o}\left(p_{3}\right)\left|U_{c o}\left(p_{1}\right)\right|^{2}}{\rho^{1}\left(p_{1}, p_{1}\right) \rho^{(1)}\left(p_{3}, p_{3}\right) \rho^{(1)}\left(p_{2}, p_{2}\right)}-\frac{\rho^{(1)}\left(p_{1}, p_{3}\right) N_{c o}^{2} U_{c o}\left(p_{1}\right) U_{c o}\left(p_{3}\right)\left|U_{c o}\left(p_{2}\right)\right|^{2}}{\rho^{1}\left(p_{1}, p_{1}\right) \rho^{(1)}\left(p_{3}, p_{3}\right) \rho^{(1)}\left(p_{2}, p_{2}\right)}\right] . \\
&
\end{aligned}
$$

that when we substitute the value of $\Lambda_{3}$ (123) into equation (6), then the corresponding expression acquires the most sophisticated shape under the assumption that the momenta of all emitted particles are identical, i.e., $p_{1}=p_{2}=p_{3}=p$. Therefore, the normalized chaotic parameter can be manipulated as

$$
w_{3}(p)=2 . \frac{\left[1+2\left[\rho_{c o}(p) / \rho(p)\right]^{3}-3\left[\rho_{c o}(p) / \rho(p)\right]^{2}\right]}{\sqrt{\left[1-\left[\rho_{c o}(p) / \rho(p)\right]^{2}\right]^{3}}} .
$$

The parameter which measures the chaotic and coherence components is called the chaotic fraction, and we define the chaotic fraction in terms of the coherence and chaotic components in order to write the normalized three particles chaotic parameter:

$$
\varepsilon(p)=\frac{\rho_{c h}(p)}{\rho_{c o}(p)+\rho_{c h}(p)},
$$

where $\rho_{c h}(p)$ and $\rho_{c o}(p)$ represent the chaotic and coherence fractions, respectively. Furthermore, the coherence fraction can be illustrated in terms of the chaos fraction as follows:

$$
\frac{\rho_{c o}(p)}{\rho_{c o}(p)+\rho_{c h}(p)}=\frac{\rho_{c o}(p)}{\rho(p)}=1-\varepsilon(p) .
$$

In particular, the special specification of the chaotic parameter can be obtained by substituting the value of equation (33) into equation (31). We can express the mathematical expression for the normalized chaotic parameter of three particles in terms of the chaotic fractions:

$$
w_{3}(p)=2 \frac{\left[1+2[1-\varepsilon(p)]^{3}-3[1-\varepsilon(p)]^{2}\right]}{\left[1-[1-\varepsilon(p)]^{2}\right]^{3 / 2}} .
$$

In addition, the normalized chaotic parameter can be simplified by applying some basic algebraic calculations, and it can be written in versatile form as follows:
From the aforementioned expression, it is obvious that the numerical value of the chaotic fraction $\varepsilon(p)=1$ for the complete chaotic sources and the normalized chaotic parameter for three particles $w_{3}(p)$ acquire the chaotic limit 2 . Similarly, the chaos fraction for the completely coherence sources is $\varepsilon(p)=0$, and it means that the value of $w_{3}(p)$ becomes zero for an ideal condition of the coherence sources but it cannot proceed to infinity in any usual case. This formula can explain the experimental data in very well way for all kind of sources which are studied in thermal as well as in mechanical engineering. We can check the consequences of such unique formula and the model results for two and three bosons which are consistent with the experimental data. The experimental results of the normalized chaos parameter can be proceeded to extract the chaotic and coherence fractions at various temperatures and momenta regimes. The derived formula is correct in all cases: (a) if a source behaves as fully chaotic and (b) the source may be partially coherent or fully coherent. Our mathematical expressions can illustrate the experimental data about two and three particles emission for the partial coherence in order to use it in the artificial intelligence.

In addition, the intercept of the normalized three particles chaotic parameter can be written in terms of the coherence fraction:

$$
I_{3}(p)=w_{3}(p)=2 \sqrt{1-f_{c o}(p)} \frac{\left[1+2 f_{c o}(p)\right]}{\left[1+f_{c o}(p)\right]^{3 / 2}} .
$$

This expression shows that if the source is completely chaotic, i.e., $f_{c o}=0$, then the intercept of $w_{3}$ attains its maximum value 2 . On the other hand, the corresponding intercept of $w_{3}$ appears to be 0 for a complete coherent source $f_{c o}=1$.

\section{Results and Discussion}

The core point of this research work is to probe the intrinsic properties of the particle production sources created by the impact of nuclei at higher energy. Statistical correlations of three particles and the corresponding normalized chaotic 

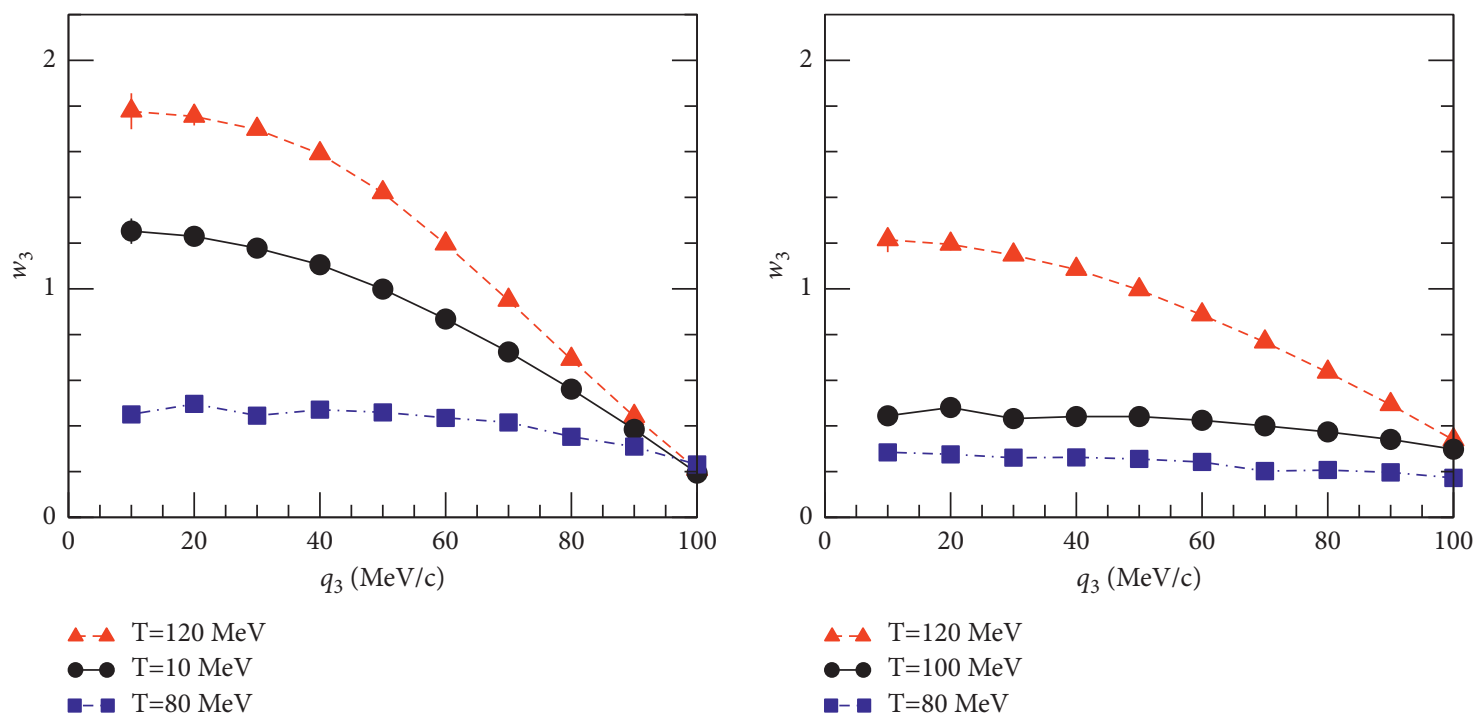

(a)

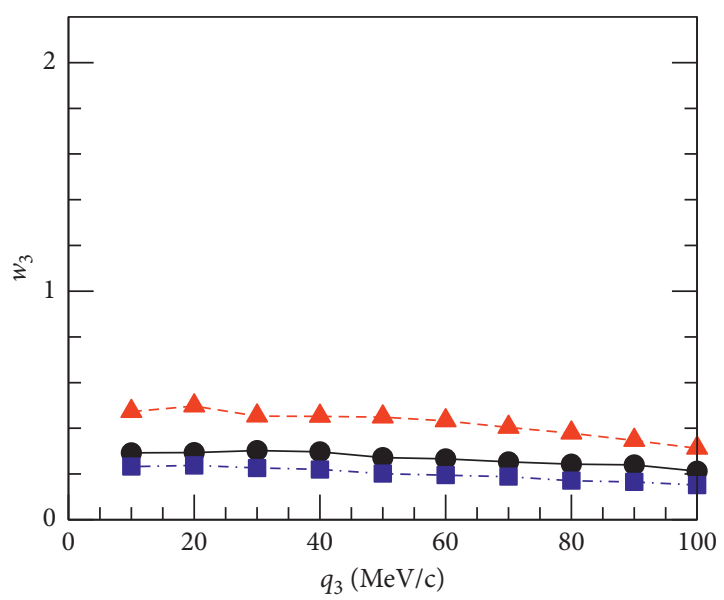

(b)

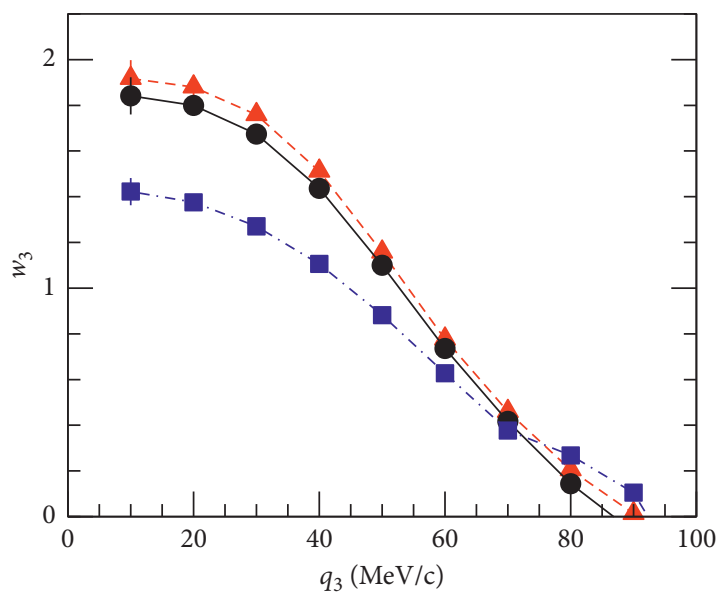

A $\mathrm{T}=120 \mathrm{MeV}$

- $\mathrm{T}=100 \mathrm{MeV}$

- $\mathrm{T}=80 \mathrm{MeV}$

A $\mathrm{T}=120 \mathrm{MeV}$

- $\mathrm{T}=100 \mathrm{MeV}$

- $\mathrm{T}=80 \mathrm{MeV}$

(c)

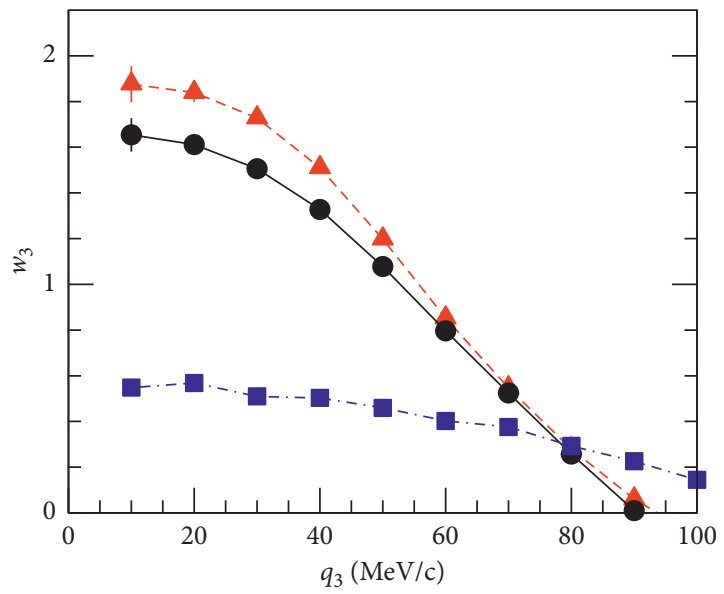

(d)

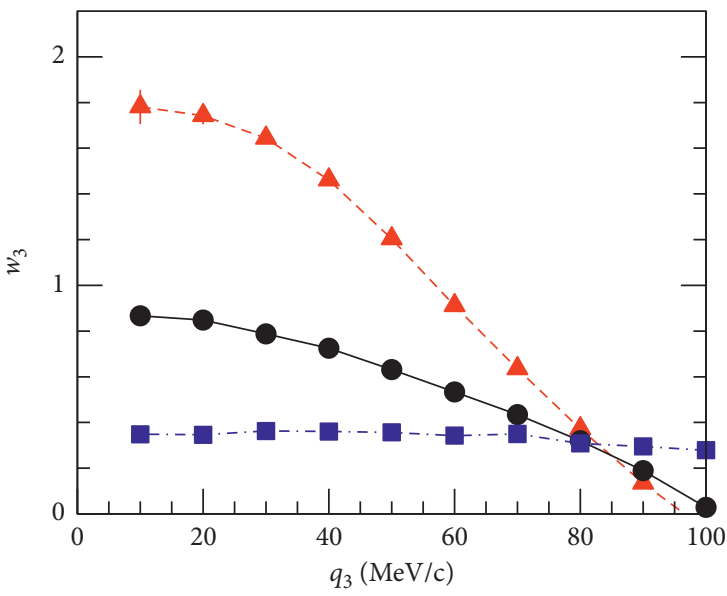

T $=120 \mathrm{MeV}$

- $\mathrm{T}=100 \mathrm{MeV}$

$\triangle \mathrm{T}=120 \mathrm{MeV}$

- $\mathrm{T}=100 \mathrm{MeV}$

- $\mathrm{T}=80 \mathrm{MeV}$

-1 $\mathrm{T}=80 \mathrm{MeV}$

(e)

(f)

FIgURE 1: Three particles normalized chaotic parameter $w_{3}$ versus $q_{3}$ at various temperatures with different particles number $N_{T}$ : (a) $K_{s}=0.35$ and $N_{T}=600 ;$ (b) $K_{s}=0.35$ and $N_{T}=800$; (c) $K_{s}=0.35$ and $N_{T}=1000$; (d) $K_{s}=0.40$ and $N_{T}=800$; (e) $K_{s}=0.40$ and $N_{T}=1000$; (f) $K_{s}=0.35$ and $N_{T}=1200$. 
parameters make it feasible consistently to quantify the temperament of the radiation sources by differential equations to use in thermal and mechanical engineering. We explicate our results about the partially chaotic source with temperature dependence characteristics and postulate that the particle ejection sources are comprised of the fluid clusters which act as the chaotic sources at a higher temperature.

Figure 1 illustrates the normalized chaotic parameter $w_{3}$ of three particles with relative momenta $q_{3}$ for the ejected sources with various temperatures $T$ and the given particles numbers $N_{T}$. $w_{3}$ for the sources possesses $N_{T}=600,800$, and 1000 for the smaller size sources of parameter $K_{s}=0.35$ as shown in Figures $1(\mathrm{a})-1(\mathrm{c})$. The chaotic parameter of the three particles suppresses considerably by increasing $N_{T}$ by fixing the temperature $T$. The reason is obvious because the plenty of particles have the probability to exist the bosons nonchaotically at lower temperature regimes. Such phenomena of temperature reduction with fixed particles $N_{T}$ reduce considerably the kinetic energy of the particles $K . E \propto T$, and the bosons continue to condensate at the lowest energy level and lead to produce the Bose-Einstein coherence which affects the bosons normalized chaotic parameter consistently. The unique results appear for small sources with $K_{s}=0.35$ due to the absence of the quantum interference, and the results approach zero in the presence of large $N_{T}=1000$ as illustrated in Figure 1(c). Furthermore, Figures 1(d) and 1(e) represent the changes of $w_{3}$ when the particle ejected sources possess excess particles with large size parameter $K_{s}=0.40$ which contain $N_{T}=800,1000$, and 1200. The chaotic parameter decreases with increasing $N_{T}$ because the source with higher particles amount acquired the large coherence effect. Such chaotic parameters increase with increasing the temperature $T$ continuously for fixing $N_{T}$ due to the reason that at extremely higher temperatures, the particles shifted at higher energy levels and the coherence effect appears negligibly. The normalized chaotic parameter approaches to its chaotic limit 2 at intercepts.

In particular, one can observe that results of $w_{3}$ are suppressed notably at the lower temperatures for the fixed $N_{T}$ under the study of both sources. The chaotic parameter of three particles shows an obvious peak with $K_{s}=0.40$ than that for small sources with size $K_{s}=0.35$ contains fixed $N_{T}$ and the temperature $T$. Such variations happen due to fact that the source with large $K_{s}$ seems to be large. The corresponding energy levels also seem to narrow which leads to pushing the maximum bosons to shift from lower to high energy states and also possess the large characteristic lengths which suppress the coherence fraction significantly.

\section{Summary and Conclusion}

The normalization of three particle cumulants to the two particles cumulant is quantified with the normalized chaotic parameter $w_{3}$. We find an obvious suppression below the chaotic limit in the measurement of $w_{3}$ at lower temperature and momentum, respectively. Such suppression appears to recommend nonchaotic components to particle production to use in thermal and mechanical engineering. It is crucial at lower temperature, and such consideration is qualitatively compatible with the origination of Bose-Einstein coherence which is contemplated to emit coherently at lower energies.

In particular, $w_{3}$ is applicable for examining the chaotic and coherence fractions at unusual energies. We evaluated $w_{3}$ at various temperatures and momentum regions and explored the consequences of the particle number and source size on the considered chaotic parameter. We have observed that the normalized three particles chaotic parameters are correlated to the chaos thermal limit of the two particle interferences and also delicate to the thermalization of the particle emissions sources.

Specifically, the possession of $w_{3}$ at temperature $T$ and the number of particles $N_{T}$ become sensitive in the high momentum regions, which contemplate the salient features of the particles emission sources from higher energy levels even the sources accompanied a substantial coherence fraction. Although the considered model explained the usual particle ejecting source which enlarged spherically, it produced the characteristics of the chaotic source and coherence fractions in the collisions. In this research work, we analyzed that the considered model confers the normalized chaotic parameter in basic agreement with the experimental applications of the chaos and coherence measurements to use in the field of thermal engineering. We realized that the chaotic parameters are suppressed crucially in the small temperature regimes at small momenta. One plausible reason for this consequence is that the production of particles from the radiated source created in the collision possesses a fraction of coherence which is nonchaotic.

\section{Data Availability}

The data are fully available without any restriction and are included within the article.

\section{Conflicts of Interest}

The authors declare that they have no conflicts of interest.

\section{Authors' Contributions}

GB conceptualized the study and wrote the original draft. WA and MS were responsible for project administration and wrote the original draft. RA and MF were responsible for resources and wrote the original draft. $\mathrm{MDF}$ and $\mathrm{NH}$ were responsible for resources and revised and edited the manuscript. IK was responsible for literature search and data analysis. Nawaf N. Hamednah and Ilyas Khan contributed equally to this work.

\section{Acknowledgments}

The authors would like to thank Prof. Wei-Ning Zhang for invigorating discussions about the consequences of the pion chaotic and coherence fractions. The authors are thankful to the Faculty of Science, Yibin University, Yibin, China, for 
support provided for this research and Yibin University for the financial support provided for this study.

\section{References}

[1] M. Gyulassy, S. K. Kauffmann, and L. W. Wilson, "Pion interferometry of nuclear collisions. I. Theory," Physical Review C, vol. 20, no. 6, pp. 2267-2292, 1979.

[2] U. A. Wienemann and U. Heinz, "Particle interferometry for relativistic heavy-ion collisions," Physics Reports, vol. 319, p. 145, 1999.

[3] R. Weiner, "Boson interferometry in high-energy physics," Physics Reports, vol. 327, no. 5, pp. 249-346, 2000.

[4] T. Csorgo, "Particle interferometry from $40-\mathrm{MeV}$ to $40-\mathrm{TeV}$," Acta Physica Hungarica, vol. 15, 2002.

[5] M. A. Lisa, S. Pratt, R. Soltz, and U. Wiedemann, "Femtoscopy in relativistic heavy ion collisions: two decades of progress," Annual Review of Nuclear and Particle Science, vol. 55, no. 1, pp. 357-402, 2005.

[6] M. M. Aggarwal, "One, two and three particle distributions from 158-A-GeV/c central $\mathrm{Pb}+\mathrm{Pb}$ collisions," Physical Review C, vol. 67, Article ID 014906, 2003.

[7] S. Pratt, "Pion lasers from high-energy collisions," Physics Letters B, vol. 301, no. 2-3, pp. 159-164, 1993.

[8] T. Csörgö and J. Zimányi, "Analytic solution of the pion-laser model," Physical Review Letters, vol. 80, p. 916, 1998.

[9] D. Gangadharan, "Techniques for multiboson interferometry," Physical Review C, vol. 92, 2015.

[10] G. Goldhaber, S. Goldhaber, W. Y. Lee, and A. Pais, "Influence of Bose-Einstein statistics on the anti-proton proton annihilation process," Physical Review, vol. 120, 1960.

[11] I. V. Andreev, M. Plumer, and R. M. Weiner, "Quantum statistical approach to Bose-Einstein correlations and its experimental implications," International Journal of Modern Physics A, vol. 8, 1993.

[12] H. Boggild, "Three pion correlations in sulphur lead collisions at the CERN SPS," Physics Letters B, vol. 455, p. 77, 1999.

[13] J. Adams, "Three pion HBT correlations in relativistic heavy ion collisions from the STAR experiment," Physical Review Letters, vol. 91, p. 262301, 2003.

[14] R. Willson, "Measurement of source chaoticity for particle emission in $\mathrm{Au}+\mathrm{Au}$ collisions at $\sqrt{s_{N N}}=130-\mathrm{GeV}$ using three particle HBT correlations," Nuclear Physics A, vol. 715, p. 619, 2003.

[15] B. B. Abelev, "Two- and three-pion quantum statistics correlations in $\mathrm{Pb}-\mathrm{Pb}$ collisions at $\sqrt{s_{N N}}=2.76 \mathrm{TeV}$ at the CERN Large Hadron Collider," Physical Review C, vol. 89, 2014.

[16] U. W. Heinz and Q. H. Zhang, "What can we learn from three pion interferometry?" Physical Review C, vol. 56, 1997.

[17] U. Ornik, M. Plumer, and D. Strottmann, "Bose condensation through resonance decay," Physics Letters B, vol. 314, 1993.

[18] J. P. Blaizot, F. Gelis, and J. Liao, "Thermalization and BoseEinstein condensation in overpopulated glasma," Nuclear Physics A, vol. 904-905, 2013.

[19] E. Ikonen, "Chaoticity parameter lamda and multiple coherent components in relativistic heavy-ion collisions," Physical Review C, vol. 78, 2008.

[20] C. Y. Wong and W. N. Zhang, "Chaoticity parameter $\lambda$ in hanbury-Brown-twiss interferometry," Physical Review $C$, vol. 76, Article ID 034905, 2007.

[21] J. Liu, P. Ru, W.-N. Zhang, and C.-Y. Wong, "Chaoticity parameter $\lambda$ in two-pion interferometry in an expanding boson gas model," Journal of Physics G: Nuclear and Particle Physics, vol. 41, no. 12, Article ID 125101, 2014.
[22] G. Bary, W.-N. Zhang, P. Ru, and J. Yang, "Analyses of multipion Bose-Einstein correlations for granular sources with coherent pion-emission droplets," Chinese Physics C, vol. 45, no. 2, Article ID 024106, 2021.

[23] M. I. Jamil, M. Arslan, and Ghani, "Lubna and others, Regioand stereoselective functionalization of alkenes with emphasis on mechanistic insight and sustainability concerns," Journal of Saudi Chemical Society, vol. 20, Article ID 101260, 2021.

[24] C. Y. Wong, W. N. Zhang, J. Liu, and P. Ru, "Chaoticity and coherence in Bose-Einstein condensation and correlations," Journal of Central European Green Innovation, vol. 4, pp. 64-79, 2016.

[25] M. Naraschewski and R. J. Glauber, "Spatial coherence and density correlations of trapped Bose gases," Physical Review A, vol. 59, no. 6, pp. 4595-4607, 1999.

[26] A. Farooqi, R. Alotaibi, T. A. Nofal, R. Farooqi, and I. Khan, "A comparative epidemiological stability analysis of predictor corrector type non-standard finite difference scheme for the transmissibility of measles," Results in Physics, vol. 21, p. 103756, 2021.

[27] B. Jamil, M. S. Anwar, A. Rasheed, and M. Irfan, "MHD Maxwell flow modeled by fractional derivatives with chemical reaction and thermal radiation," Chinese Journal of Physics, vol. 67 , pp. 512-533, 2020.

[28] A. Khan Zareen and H. Ahmad, "Qualitative properties of solutions of fractional differential and difference equations arising in physical models," Fractals, vol. 7, 2021.

[29] Y. Karaca, M. Moonis, and D. Baleanu, "Fractal and multifractional-based predictive optimization model for stroke subtypes' classification," Chaos, Solitons \& Fractals, vol. 136, Article ID 109820, 2020.

[30] A. Abdon and A. Seda İğret, "New concept in calculus: piecewise differential and integral operators," Chaos, Solitons \& Fractals, vol. 145, Article ID 110638, 2021.

[31] M. S. Hashemi, "A novel approach to find exact solutions of fractional evolution equations with non-singular kernel derivative," Chaos, Solitons \& Fractals, vol. 152, Article ID 111367, 2021.

[32] M. S. Hashemi, M. Inc, and A. Yusuf, "On three-dimensional variable order time fractional chaotic system with nonsingular kernel," Chaos, Solitons \& Fractals, vol. 133, Article ID 109628, 2020.

[33] J. Hadi, O. Orozco-López, and M. Pacheco, "Simulation and experimental validation of a non-equilibrium chaotic system," Chaos, Solitons \& Fractals, vol. 143, p. 110539, 2021.

[34] X. Pei-Ying, J. Hadi, and A. Raúl, "Spectral entropy analysis and synchronization of a multi-stable fractional-order chaotic system using a novel neural network-based chattering-free sliding mode technique," Chaos, Solitons \& Fractals, vol. 144, Article ID 110576, 2021.

[35] M. S. Anwar, R. T. M. Shahzad, M. Irfan, and M. Z. Ashraf, "Electrified fractional nanofluid flow with suspended carbon nanotubes," Computers \& Mathematics with Applications, vol. 80 , no. 5 , pp. 1375-1386, 2020. 